\title{
Realistic limits for subpixel movement detection
}

\author{
David Mas, ${ }^{1,}{ }^{*}$ Jorge Perez, $^{1}$ Belen Ferrer, $^{2}$ Julian Espinosa $^{1}$ \\ ${ }^{1}$ Inst. Univ. Física Aplicada a las Ciencias y Tecnologias. Universidad de Alicante, P.O. Box 99, 03080, Spain \\ ${ }^{2}$ Dep. Ing. Civil, Universidad de Alicante, , P.O. Box 99, 03080, Spain \\ *Corresponding author: david.mas@ua.es
}

Received XX Month XXXX; revised XX Month, XXXX; accepted XX Month XXXX; posted XX Month XXXX (Doc. ID XXXXX); published XX Month XXXX

\begin{abstract}
Object tracking with subpixel accuracy is of fundamental importance in many fields since it provides optimal performance at relatively low-cost. Although there are many theoretical proposals that lead to resolution increments of several orders of magnitude, in practice, this resolution is limited by the imaging systems. In this paper we propose and demonstrate through simple numerical models a realistic limit for subpixel accuracy. The final result is that maximum achievable resolution enhancement is connected with the dynamic range of the image, i.e. the detection limit is $1 / 2^{\wedge}$ (nr.bits). The results here presented may help to proper design of superresolution experiments in microscopy, surveillance, defense and other fields. (C) 2016 Optical Society of America
\end{abstract}

OCIS codes: (100.6640) Superresolution; (100.4999) Pattern recognition, target tracking (040.7290) Video, (170.0110) Imaging systems.

http://dx.doi.org/10.1364/AO.99.099999

\section{Introduction}

Object detection and tracking is a common operation in many tasks like surveillance, security, acoustics or civil engineering. Although other systems can be used, the most common approach is through vision systems and image analysis. Unfortunately, and despite their continuous improvement in temporal and spatial resolutions, these devices are still considered as low-resolution systems mainly in those applications concerning video acquisition for assessing dynamic processes [1].

In the last years, hardware improvements in both sensors and storage capabilities have increased the temporal resolution of video systems. Nevertheless, at constant data flux, increasing the temporal resolution implies reducing the spatial resolution. Therefore many systems provide high video rates with limited image quality, thus impeding the use of those cameras for applications where both amplitude and temporal accuracy are needed. Notice that even the most advanced high-speed cameras rarely use sensors larger than $10 \mathrm{Mpx}$ and this at a huge cost, while, for static cameras, sensors larger than 25 Mpx are easy to find.

This limited spatial resolution encourages the search of processes that may overcome these limits and provide enhanced capabilities. Subpixel techniques allow increasing the image resolution of the digital detectors and thus, improving the performance of existing devices at a reasonable computational and economical cost. Therefore, defining the actual experimental limitations of subpixel resolution is of the outmost importance to explore the potential applications of image and video systems.

Object tracking with subpixel resolution depends on whether we are referring to a target that describes a known trajectory or to absolute detection of movement, with no a priori knowledge about the system. In the first case tracking resolution can be very much enhanced from fitting to a trajectory and interpolation. In the second case there are no external information that could guide the detection and decrease the positioning errors. Therefore, the expected accuracy of absolute detection is much lower. In this paper we will focus in the second case.

Theoretical models propose target designs that allow absolute object tracking with virtually infinite resolution. In [2], the authors propose different target shapes with binary images. In [3], the authors revisited the idea and show that, by using convenient targets, the theoretical maximum accuracy is of the order of $1 / \mathrm{N}$ px, being $\mathrm{N}$ the total number of pixels in the scene. Although these targets are of difficult implementation, they may be useful to analyze the efficiency of different implementations and even to design new methods [4].

Nevertheless, and despite the theoretically achievable improvement, to the best of our knowledge, experimental methods rarely reached absolute shifts detections smaller than $0.01 \mathrm{px}$. which is far of the theoretical limits. Noise and inaccuracies in the sensor can degrade the signal and thus reduce the optimal accuracy in one or two order of magnitude but even so, experimental and theoretical predictions are still too much different.

In this paper, we propose a simple method to determine the maximum accuracy that can reasonably be expected in target tracking tasks. Our goal is to provide a rule of thumb that may help to optimize the experimental implementations of object tracking methods and show a realistic limit to the accuracy enhance. The imaging system will be considered here as a black box that connects the object with an output image in gray levels. In this approximation, errors coming from the optics, camera or preprocessing algorithms will be considered as a whole and experimentally estimated.

Our main hypothesis is that the necessary condition for movement detection is that a single pixel changes its status. Therefore, we reduce 
the problem to analyze the main factors that can provoke this pixel change.

As first approximation we will consider that only a movement can change a pixel status. Then we will consider the pixel as a simple light container and we will propose simple models on how the content of the container may vary with the object movement. With this, a relation between the tracking accuracy and the image dynamic range is obtained. Finally, we will consider the main distorting factors that may alter the image response and are not connected to object movement and a brief discussion about their effect on the final accuracy will be presented.

Our proposal is completed with numerical simulations and an experimental implementation that illustrate some of the concepts here described. Finally, the main conclusions are summarized.

\section{Proposal}

As we stated above, according to what is established in [4], special objects can be tracked with accuracy of the order of $1 / \mathrm{N}$. This affirmation was proved through numerical simulations and with different error criteria. Unfortunately, the physical limitations of the sensors and the particular conditions imposed on the targets clearly limits the accuracy and real implementations will not be so sensitive. In what follows, we will try to determine which is the ultimate experimental limiting factor in the design of subpixel tracking methods.

First of all, we will assume some convenient boundary conditions, although they are not really limiting for majority of applications.. With respect to the lenses we suppose that they are free of aberrations, back reflections or scattering and paraxial optics is applicable in the main region of the sensor. This means that the movement in the object and image planes is proportional, so we can reduce our description to shifts the image-sensor plane. Regarding the sensor, we consider that its fill factor is $100 \%$, so the active area between two adjacent pixels is continuous. This is not a very strong limitation, since last generations of CCD and CMOS implement efficient lens arrays that avoid the inter-pixel spacing [5]

Additionally, let us suppose by the moment that the system is free of noise and the detector is not saturated. We also assume that our sensor is monochromatic and linear so the response only depends on the luminance. Again, this is not a severe assumption since most detector behave in this way under standard lab conditions.

Common gray-scale image formats linearly rescale the response between 0 (black) and 1 (white) in quantized levels. For a sensor of $B$ bits per pixel, the number of allowable levels is $2^{\mathrm{B}}$. The most known cameras implement sensors of 8 bits/pixel allowing the registration of 256 grey levels per pixel.

With independence of the algorithm used to movement detection, the necessary condition for movement detection is that one single pixel changes its status. Therefore, and without losing generality, let us reduce our problem to analyze this single pixel, wherever it is located.

Let us assume that the object, in the scene is shifted an arbitrary small distance in the $x$-direction. The image, in the sensor plane will also move an equivalent distance $\delta x$. Due to the quantization of the energy levels a change in the test-dot will only be detected when the energy increase reaches one of the upper/lower quantization steps, i.e. the positive or negative energy variation is an integer multiple of $1 / 2^{\mathrm{B}}$.

Detection of displacement $\delta x$ is linked to the capability of detecting the energy transfer from a pixel detector to the adjacent one, and this depends on the energy profile so a general rule is difficult to establish. Therefore, we will propose two basic models. The simplest one considers that the PSF (Point Spread Function) has a rectangular profile, with a flat top level and an abrupt decay. The second model consists of taking a more realistic Gaussian PSF profile.
Let us start with the squared flat energy profile and how this model predicts the energy transfer from one pixel to the adjacent one: being $E$ the energy of the squared profile and $p$ the side length of the pixel sensor, the normalized response is

$$
\boldsymbol{E} \times \boldsymbol{p} \times \boldsymbol{p}=\mathbf{1}
$$

If the object moves laterally, part of this squared profile will enter the adjacent sensor and the detection will be possible when the following condition is fulfilled:

$$
E \times p \times \delta x \geq \frac{1}{2^{B}}
$$

Or, according to equation (1):

$$
\delta x \geq \frac{p}{2^{B}} \quad \rightarrow \quad \frac{\delta x}{p} \equiv \delta p \geq \frac{1}{2^{B}}
$$

where, in the expression at the right, detection accuracy is expressed in pixel units. According to this, for the most typical case of 8-bits cameras, the minimum movement that is detected is $\delta p=1 / 256 \cong 0.004 \mathrm{px}$. For cameras of 10,12 bits, the detection limit is of the order of $1 \mathrm{e}-3$ and $2 \mathrm{e}-4$ respectively. Notice that this rule cannot be extended to 24 bit color cameras since they use three different channels of 8 bits each one. Each of these channels is captured by different pixels and the final image is composed by software [6].

This result can be refined by using a more realistic energy profile model. Assuming a monochromatic coherent illumination and a diffraction-limited system with a circular aperture, the intensity profile of a single dot image (PSF) takes the form of an Airy disk, which may be approached by a Gaussian function [7]:

$$
I(\rho)=I_{0}\left(\frac{2 J_{1}(k N A \rho)}{k N A \rho}\right)^{2} \approx I_{0}^{\prime} \exp \left(\frac{-\rho^{2}}{2 \sigma^{2}}\right)
$$

being $I_{0}$ the maximum intensity of the pattern at the Airy disk center, $J_{1}$ the first-order Bessel function of the first kind, $k=2 \pi / \lambda$ is the wavenumber, $\rho$ the radial distance from the center of the spot to any point in the image plane and $N A$ is the numerical aperture of the optical system.

If we impose that $I_{0}=I^{\prime} \circ$ and find the value of $\sigma$ that gives the optimal approximation to the airy pattern, we obtain:

$$
\sigma \approx 0.21 \frac{\lambda}{N A}
$$

As we did above, let us reduce our problem to a single dot focused onto a single pixel. In this case, the normalized energy response will be:

$$
\int_{-\frac{p}{2}}^{\frac{p}{2}} I_{0} \exp \left(-\frac{x^{2}}{2 \sigma^{2}}\right) d x \equiv \int_{-\frac{p}{2}}^{\frac{p}{2}} I_{0} \exp \left(-\frac{N A^{2}}{0.0882 \lambda^{2}} x^{2}\right)=1
$$

A displacement of $\delta x$ in the spot will transfer part of the energy from one pixel to the adjacent one, i.e.:

$$
\begin{aligned}
\int_{-\frac{p}{2}+\delta x}^{\frac{p}{2}} I_{0} \exp \left(-\frac{N A^{2}}{0.0882 \lambda^{2}} x^{2}\right) d x+ \\
+\int_{\frac{p}{2}}^{\frac{p}{2}+\delta x} I_{0} \exp \left(-\frac{N A^{2}}{0.0882 \lambda^{2}} x^{2}\right) d x=1
\end{aligned}
$$

As we said before, detection will only happen when the energy in the new pixel surpasses a minimum threshold, thus giving a condition for the minimum displacement $\delta x$ needed for detection:

$$
E_{t h}=\int_{\frac{p}{2}}^{\frac{p}{2}+\delta x} I_{0} \exp \left(-\frac{N A^{2}}{0.0882 \lambda^{2}} x^{2}\right) d x \geq \frac{1}{2^{B}}
$$

Conditions in expressions (6) and (8) can be numerically evaluated through the error function erf(x), defined as: 


$$
\operatorname{erf}(x)=\frac{2}{\sqrt{\pi}} \int_{0}^{x} \exp \left(-t^{2}\right) d t
$$

thus giving the expression:

$$
E_{\text {th }} \cong \frac{1}{2}\left[\frac{\operatorname{erf}\left[\frac{N A}{0,297 \lambda}\left(\frac{p}{2}+\delta x\right)\right]}{\operatorname{erf}\left[\frac{N A}{0,297 \lambda} \frac{p}{2}\right]}-1\right]
$$

Evaluation of expression in (10) requires the selection of a proper wavelength $\lambda$, pixel size, $p$ and numerical aperture, $N A$. Nevertheless, since we are just giving an order of magnitude of the minimum shift needed for subpixel detection, we can state additional boundary conditions.

The Rayleigh criterion establishes the minimum distance between two Airy disks so that they can be distinguished. In an optimized design, this criterion should be matched by the resolution limit of the discrete sensor, which is limited by the Nyquist limit. According to this, we can affirm that the minimum distance between two spots must be at least equal to two pixels:

$$
\frac{0.61 \times \lambda}{N A}=2 \times p
$$

Therefore, if the optical system is perfectly tuned with the sensor and is capable to produce images at the Nyquist limit, we can use equation (11) to obtain a relation between the three variables implied in (10) so the expression is very much simplified:

$$
E_{t h} \cong 0,9394 \operatorname{erf}(0,5135+1,027 \delta p)-0,5
$$

being $\delta p=\delta x / p$. Notice that this expression only depends on the ratio between the displacement and the pixel size, or equivalently, on the fraction of pixel displaced.

In figure 1, we represent the values fulfilling equation (12) in front of the values of the threshold obtained for the simplified case in equation (3). We see that the difference between them approximately is a multiplicative factor. Therefore, the realistic model shows less resolution than the simplified one, but can be considered equivalent in terms of order of magnitude.

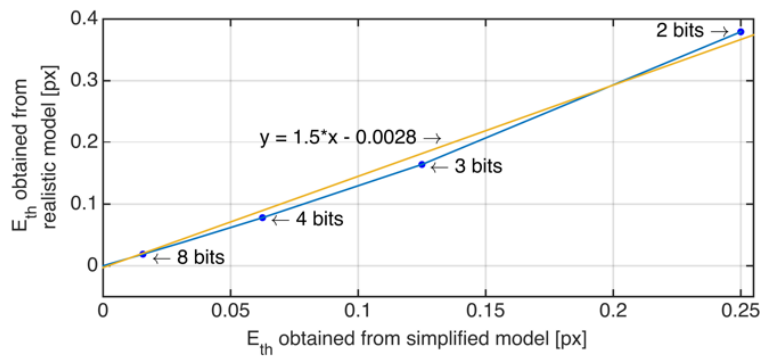

Figure 1. Comparison between detection thresholds $E_{\text {th }}$ obtained from the simplified model in equation (3) and a more realistic model, in equation (12). A linear fitting has been added in order to clarify the trend.

This model considers a single pixel of an extended image. Real objects may have complex shapes and energy profiles so depending on the particular energy profile it may happen that the round-off error makes that shift-detection from some initial positions is more accurate than from others. In some cases, mainly in complex objects, the original intensity value may be closer to the detection threshold and therefore a small change may raise the signal into the new pixel so detection may happen with a shift smaller than that obtained above. This idea has been already explained in [3], and some applications have been proposed in $[4,9]$.
Statistically, the above described situation may happen for half the dots of the image. When a shift is produced, the pixels whose incoming energy is the upper half part of the quantization step will be first detected with only half the needed displacement. Once all these pixels have been detected their energy is at the lower part of a new quantization step while the remaining ones will have their energy in the upper part so the situation is repeated. As a consequence of this, one could further increase, by a factor 2 the limit previously established, i.e.

$$
\delta x \gtrsim \frac{p}{2^{B+1}} \quad \rightarrow \quad \delta p \gtrsim \frac{1}{2^{B+1}}
$$

Deduction of expression (13) has been done by considering a "perfect" system that is free of aberrations and noise. For the majority of applications, the first requirement is easy to meet with quality optics and within certain restrictions such as correct alignment, paraxial region and precise focusing. Therefore, we will not further develop this issue.

Unfortunately, the second requirement -absence of noise- is much difficult to fulfill. Noise in the image may come from a wide variety of sources. Some are inherent to the sensor and electronics and include electronic and thermal inaccuracies, different pixel response through the CCD/CMOS area or vibrations transmitted to the camera due to an inadequate mechanical isolation. There may also be other error sources coming from the object. These include changes in the illumination, or in the transmitting medium.

Analysis of the different errors affecting to the accuracy of image acquisition is really complex and it is outside the scope of this manuscript. As we said above, our goal is to provide a rule of thumb to determine the expected accuracy that could be used for non-image experts. An accurate analysis about subpixel object location and error sources can be found in [10].

Independently of the error source, one can experimentally estimate the error of the setup by capturing a sequence of the static target, i.e., before the movement starts, and perform a tracking. Since the object is static, all movement detected may come from the noise or from the calculation method itself. In our experience, the standard deviation of the calculated positions is a good estimator of the positioning error due to noise. When the target starts its movement, one can affirm that the target has been shifted only when its detected position is above the level of noise. According to this, an oscillation of $1 / 256$ or equivalently 0.004 px will increase the threshold displacement needed to distinguish the movement, thus diminishing the expected tracking accuracy. This method was used in [11] to compare the subpixel accuracy between a low-cost camera and a medium level camera.

For absolute and precise detection of movement one can fix the error threshold in three times the standard deviation thus almost discarding the possibility of a false movement detection. If the target is following a trajectory along a known path, this requirement can be relaxed, since other criterions may be used to determine the accuracy of the tracking.

\section{Numerical implementation}

In order to demonstrate our proposal, we have numerically implemented some examples of movement detection after subpixel displacement. For the implementation, we have taken different onedimensional profiles.

Two objects have been analytically generated and evaluated on a vector of $N=512 p x$, with the variable ranging in the interval $x \in[-N / 2, N / 2-1] p x$. A shape factor modeling the object width $L \in[10,200]$ $p x$ has also been introduced in both cases so that we can account the object profile influence. For each shape factor, the object has been displaced a total length of one pixel in steps of $0.0001 p x$ which is one order of magnitude above the highest expected resolution. Both the original and the displaced functions have been quantized to a different 
number of bits and the difference between them is evaluated. The total number of steps needed for producing a detectable change is accounted and with this, the detection resolution is achieved. The average resolution along one full path is obtained for each shape and quantization level. Tests programs have been implemented in Matlab and can be downloaded and checked from Ref. [9]

The first object used has been a Gaussian profile, with equation:

$$
f_{L}(x)=\exp \left[-\frac{x^{2}}{(L / 2)^{2}}\right]
$$

In Figure 2 we represent the results obtained according to the procedure explained above. We have used the logarithmic scale in order to allow better visualization. We can see there that, for large quantization steps ( 2 bits), the shape of the object influences the error. The quantization effect explained before is very strong and the calculated threshold may be lower than the one established by our models. As the quantization levels become narrower, the shape factor is less and less relevant and the detection threshold is less dependent of the object shape. Notice also that all threshold values calculated are around the values predicted in (13).

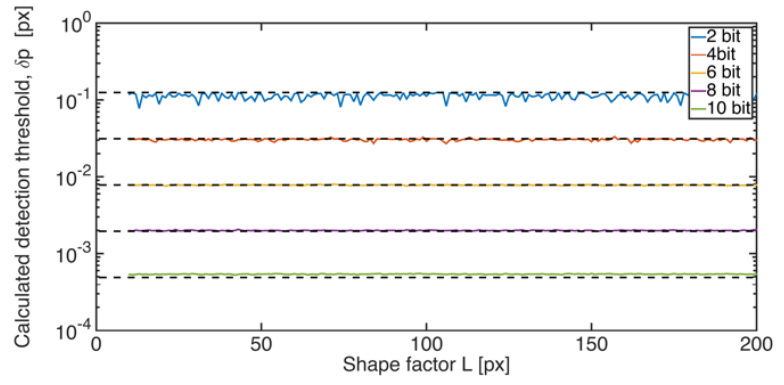

Figure 2: Calculated detection threshold or a Gaussian profile with different shape factors as defined in equation (14). Dashed lines represent the values in equation (13).

The next object implemented is a triangular shape with base $L$ and height equal to one:

$$
f(x)= \begin{cases}\frac{2}{L} x+1 \quad \text { for } x \in[-\mathrm{L} / 2,0] \\ -\frac{2}{L} x+1 \text { for } x \in[0, \mathrm{~L} / 2] \\ 0 \quad \text { otherwise }\end{cases}
$$

Notice that this function includes three non-derivable points whose discretization will be problematic. The threshold needed for movement detection is depicted in figure 3 .

There we can see that, for the majority of the shapes and quantization levels, the detection threshold coincides with the theoretical value in equation (13), in accordance with the explanation here given. Nevertheless, we can notice that some particular values show important and localized peaks. Since we are representing the threshold average for a full pixel displacement, this means that subpixel resolution is drastically decreased for this particular shape. This situation occurs always with objects with straight borders and sharp corners and was already addressed in [10]. In brief, this effect happens when the lines directions are geometrically linked to the squared detector matrix and the quantization steps are too large. In this case, the round-up effect introduces large errors that are not statistically compensated since the pixel distribution on the image plane is highly anisotropic. This strange behavior tends to disappear as the quantization steps are more and more narrow.

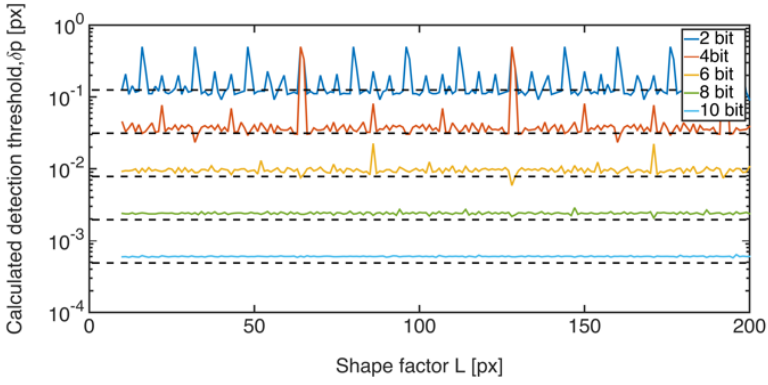

Figure 3: Calculated detection threshold or a Gaussian profile with different shape factors as defined in equation (15). Dashed lines represent the values in equation (13).

Fortunately, this only happens in very specific situations, shapes and quantization levels. Moreover, real objects are rarely composed by straight lines but present more complex shapes so, in the large majority of cases, the rule for realistic subpixel accuracy here established remains valid.

We would like to focus the reader's attention on the fact that, in both cases represented in figures 2 and 3 , as the quantization step becomes shorter (more bits used) the detection threshold is a bit lower than the one predicted by the equation (13). Up to our knowledge this happens because the dynamic range tends to be continuous and the round-up effect is less and less important. In the limit, this division between upper and lower half of the step does not have sense and the threshold will converge to the value in equation (3).

\section{Experimental test}

In order to check the validity of our estimations, an experimental test has been carried out. A simple object has been printed and attached to a continuous linear motorized stage (DDSM100/M from Thorlabs). The platform will horizontally displace the object a total distance of $3 \mathrm{~mm}$ at a speed of $0.3 \mathrm{~mm} / \mathrm{s}$. The minimum incremental movement is $500 \mathrm{~nm}$ and the repeatability is of $\pm 1.5 \mu \mathrm{m}$. The scene was illuminated by two halogen lamps connected to a stabilized DC generator, in order to avoid light oscillation from the general AC installation.

The movement was recorded with an AOS X-PRI camera situated at $5 \mathrm{~m}$ from the object. The spatial and temporal resolution of the camera are $800 \times 600 \mathrm{px}$ and 200 frames per second. With this configuration, the conversion rate is $2.4012 \mathrm{~mm} / \mathrm{px}$, and the displacement of the target image on the sensor plane between frames is $0.0013 \mathrm{px}$. Since the camera works at 8 bits, the movement between two consecutive frames is below the best expected accuracy. Operation of the camera and the stage was done remotely in order to avoid vibrations.

The object used as a target was a 2D Gaussian function. The target width on the image plane has been estimated in around $50 \mathrm{px}$. The Gaussian figure was printed in negative but after the capture was again inverted in order to obtain a white object against a black background. In figure 4 we show the scene as it is captured by the camera together with a zoom of the object with its luminance levels already inverted.

Object tracking may be strongly influenced by the algorithm used. In our case, the position of the target was tracked though its centroid, which was calculated using the Crocker and Grier's algorithms [13]. We used the MATLAB implementations proposed by the authors in [14], conveniently adapted to our inputs. We would like to emphasize that the calculations have been performed without pre-processing for image enhancement or noise reduction, which means that the error obtained by this experiment could be lowered.

As we said in Section 2, a sequence of the static object was captured in order to estimate the error of the method, which includes the 
experimental setup and the accuracy of calculations. Prior to that step a detection test over a synthetic scene was performed in order to check that the numerical error introduced by the processing algorithm is, at least one order of magnitude below the best resolution experimentally achievable according to expression (13). The analysis of a sequence of 1 second (200 frames) provide as standard deviation of $0.0038 \mathrm{px}$, which is in the order of 1 luminance level.

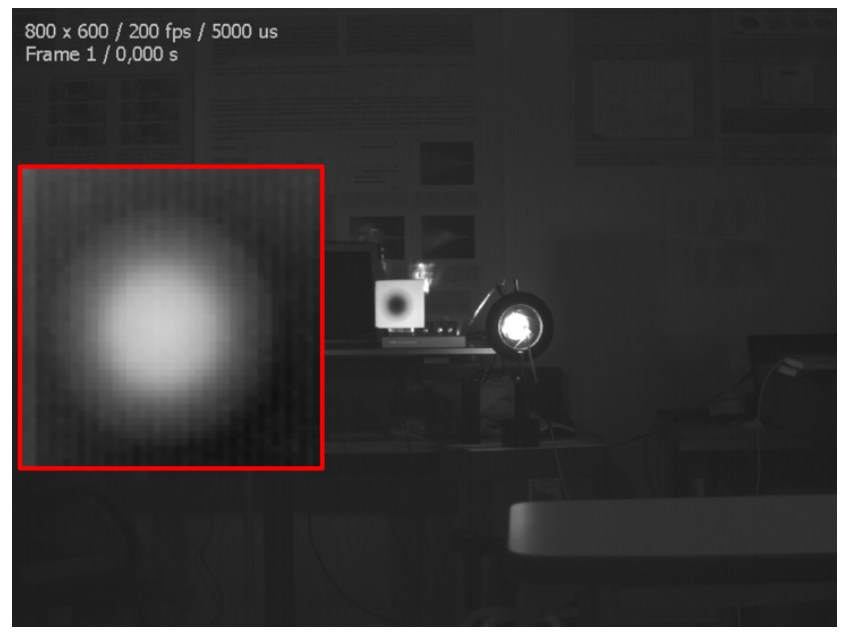

Figure 4: Complete scene as it was captured by the AOS camera. The red square shows a zoom of the target. Notice that target gray levels have been inverted in order to facilitate processing and tracking and frame by frame.

The representation of the position of the moving target versus time provides a straight line whose slope coincides with the target speed. The positioning error has been analyzed by comparing the obtained position with the real one theoretically calculated. As a global estimator, we use the standard deviation of the error. In the case we are analyzing, this value is of 0.0066 , almost double than the static error., as predicted in Section 2

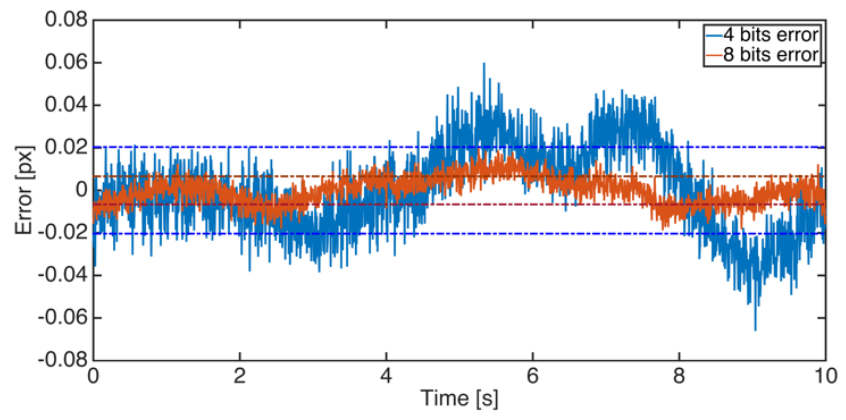

Figure 5: Location error for the object in Figure 4 moving horizontally. Dashed blue lines represent the standard error of the 4 bits sequence, while dashed red lines represent the standard error of the 8 bits sequence.

Images from the camera have been manipulated so that a realistic simulation for 4 bits could be obtained. In this case, the obtained error for the method (static target) has been 0.0126 , while for the moving target, the standard deviation of the error has been 0.0203 . In Figure 5 the error obtained for both 8 and 4 bits is depicted.

Notice that the error obtained for the 8 bits case is of the order of magnitude predicted by equations (3) and (13) although a bit higher because the experimental errors. In the 4 bits example, the order of magnitude is the same, but the value is lower than expected, so the accuracy is higher. Thus result must be taken with care since the image is not directly obtained in 4 bits but manipulated. Therefore, the noise has been probably lost in the rounding process, thus giving a false impression of accuracy.

Finally notice that the results here obtained have been calculated without noise reduction or any other image manipulations. Wise image processing could further increase the accuracy of the method.

\section{Conclusion}

Summarizing all our discussion, we have stated here that under ideal experimental conditions, absolute detection of object shift can be improved up to a factor equal to the inverse of the number of gray levels in the image. Object energy profile or image preprocessing may increment this accuracy but, real-life conditions, including realistic energy profiles, noise and aberrations, may decrease the accuracy. Therefore, our conclusion is that equation (3) is a safe estimation of an achievable subpixel accuracy.

We would like to underline that this does not mean that resolution can be arbitrarily increased with the number of bits. In [3] the authors show that, in absence of other limitations, the object geometry also imposes a severe limit, although much higher than the one here obtained. Notice that the limit obtained is inherent to the digitization process and does not depend on the detection algorithm so it is applicable in almost all tracking algorithms.

Our affirmation has been tested through numerical examples. We have implemented different objects with different shapes and quantization levels and we have shown that our proposal stands in all cases, provided that the object shape is complex enough. An experimental test has been also carried in order to check the accuracy of our predictions and gives some hints about the error estimation.

Results here presented may be very useful for researchers working in video processing for target tracking, mainly in microscopy where resolution increases are of the utmost importance. On the one hand, our proposal clearly states the limits of the maximum resolution enhancement that can be reached with a given system. On the other hand, it provides a reference value to push non-optimal systems to the maximum achievable resolution that can be obtained with a given experimental setup, with independence of image processing techniques.

Funding Information. Generalitat Valenciana through the projects PROMETEOII/2015/015 and GV/2015/116 and the University of Alicante through the project GRE13-10.

\section{References}

1. B. Ferrer, J. Espinosa, J. Pérez, S. Ivorra, D. Mas “Optical scanning for structural vibration measurement" Res. Nondestruct. Eval, 22, 61-75 (2011)

2. A. M. Bruckstein, L. O'Gorman, A. Orlitsky “Design of shapes for precise image registration," IEEE Trans. Inform. Theory, 44, 3156-3162 (1998).

3. D. Mas, B. Ferrer, J. T. Sheridan, J. Espinosa "Resolution limits to object tracking with subpixel accuracy" Opt. Lett, 37,4877-4879 (2012)

4. B. Ferrer, J. Espinosa, A.B. Roig, J. Perez, D. Mas, "Vibration frequency measurement using a local multithreshold technique" Opt. Express, 21, 26198-26208 (2013)

5. See for example [retrieved 21 March 2016] http://www.framos.com/products/en/sensors.html or http://www.hamamatsu.com/jp/en/4206.html

6. See for example [retrieved 21 March 2016] https://en.wikipedia.org/wiki/Charge-coupled device 
7. B. Zhang, J. Zerubia, J. C. Olivo Marin, "Gaussian approximations of fluorescence microscope point spread function models" App. Opt., 46, 1819-1829 (2007)

8. D. Mas, B. Ferrer, P. Acevedo, J. Espinosa "Methods and algorithms for video-based multi-point frequency measuring and mapping" Measurement, 85, 164-174 (2015)

9. D. Mas. "Matlab code for calculation of shift detection threshold"(2016) [retrieved 21 March 2016] http://hdl.handle.net/10045/53726

10. C. Zhai, M. Shao, R. Goullioud, B. Nemati, "Micro-pixel accuracy centroid estimation and detector calibration" P Roy Soc A-Math Phy 476, 3550$3569(2011)$

11. D. Mas, J. Espinosa, A.B. Roig, B. Ferrer, J, Perez, C. Illueca, "Measurement of wide frequency range structural microvibrations with a pocket digital camera and sub-pixel techniques" Appl. Opt, 51, 2664-2671 (2012).

12. J. Espinosa, J. Perez, B. Ferrer, D. Mas "Method for targetless tracking subpixel in-plane movements" Applied Optics, 54, 7760-7765 (2015)

13. J. C. Crocker, D. G. Grier "Methods for digital video microscopy for coloidal studies" J. Colloid. Interf. Sci., 179, 298-310 (1996)

14. D. Blair, E. Dufresne "The Matlab tracking code repository" [retrieved 26 May 2016] http://site.physics.georgetown.edu/matlab/ 